\title{
Caracterización de los diferentes tipos de reacciones adversas relacionadas con el uso de medicamentos en un hospital de segundo nivel de complejidad de Itagüí, Antioquia
}

\author{
Characterization of the various types of \\ adverse reactions related to drug use in a \\ secondary-care hospital in Itagüí, Antioquia
}

Deisy Yuliana García Uribe ; Alexandra María Ortega Henao

Tutores: Isabel Escobar²; Erika Francisca Garrido Zea

Médico, Facultad de Ciencias de la Salud, Corporación Universitaria Remington, calle 51 \# 51-27, Medellín, Colombia.

2 Médica y Cirujana, especialista en Toxicología clínica, docenteFacultad de Ciencias de la Salud, Corporación Universitaria Remington, calle 51 \# 51-27, Medellín, Colombia. Correo: isaescobartoledo@gmail.com

3 Microbiológa, MSc Biología, docente investigadora Facultad de Ciencias de la Salud, Corporación Universitaria Remington, calle 51 \# 51-27, Medellín, Colombia.
Resumen

El uso concomitante de diversos fármacos es un proceso que se realiza frecuentemente en la práctica médica, dado que, a menudo nos enfrentamos con pacientes que presentan afecciones orgánicas simultáneas que deben tratarse con varios medicamentos. Al utilizar estos agentes terapéuticos de forma conjunta se presenta la disyuntiva de saber si la combinación terapéutica realizada entre un solo grupo de medicamentos o varios grupos de estos, se está ejecutando de forma racional o irracional en defensa o en contra del estado de salud de un paciente y más aun cuando se conoce que aproximadamente el $50 \%$ de las prescripciones que se realizan a diario, conllevan al menos a una forma de interacción medicamentosa. Las Reacciones Adversas a Medicamentos (RAM) causan una considerable morbilidad y mortalidad en todo el mundo y en muchos casos son evitables. Una parte importante de la patología iatrogénica que afecta a la población puede haber sido consecuencia de la prescripción irreflexiva de medicamentos innecesarios, polifarmacia o que no eran de primera elección para tratar su patología actual. El presente trabajo tiene por objetivo caracterizar los diferentes tipos de reacciones 
adversas relacionadas con el uso de medicamentos en un hospital de segundo nivel de complejidad en Itagüí, Antioquia; para alcanzar dicho objetivo, se recurrió a la revisión de los registros del programa de farmacovigilancia de la institución hospitalaria llevados a cabo por químicos farmacéuticos y la médica toxicóloga. El estudio encontró que la mayoría de las RAM serían evitables, si se disminuyera la automedicación y la polimedicación, lo que se puede conseguir con una buena educación al paciente. Las RAM no prevenibles se deben a procesos inmunológicos, los cuales no son controlados por el personal médico, ya que dependen de cada individuo.

Palabras clave: farmacoterapia, reacciones medicamentosas, interacciones medicamentosas.

\section{Abstract}

The concomitant use of several drugs is a common process in medical practice, since medical professionals often deal with patients with simultaneous conditions that must be treated with multiple medications. When combining these therapeutic agents, practitioners are confronted with the dilemma of whether the therapeutic combination, either of medications within one group of drugs or of several drug groups, is being rationally or irrationally conducted, in defense or against the state of the health of a patient. This is even more so when it is known that about $50 \%$ of daily prescriptions involve at least one form of drug interaction. Adverse drug reactions (ADRs) are a significant cause of morbidity and mortality worldwide which in many cases is preventable. A significant proportion of iatrogenic diseases that affect the population may have been a result of careless prescription of unnecessary drugs, polypharmacy or the prescription of drugs that were not the first choice for the relevant condition. This paper aims to characterize the different types of adverse reactions related to drug use in a secondary-care hospital in Itagüi (Antioquia). To achieve this goal, the records associated to the hospital pharmacovigilance program, conducted by pharmaceutical chemists and a toxicologist, were examined. The study found that most DRAs could be avoided by reducing selfmedication and polypharmacy, which can be achieved through effective patient education. Non-preventable DRAs are linked to immunological processes, which are not controlled by the medical personnel, as they vary with each individual.

Keywords: drug therapy, drug reactions, drug interactions.

\section{Introducción}

Como consecuencia de la epidemia de focomelia en recién nacidos causada por la talidomida en Europa a partir de 1960, varios países emprendieron una incipiente vigilancia de los medicamentos. En 1968, la Organización Mundial de la Salud (OMS), en el marco del Programa Internacional para el Monitoreo de Medicamentos, propuso la creación de un centro para la farmacovigilancia internacional, establecido actualmente en Uppsala, Suecia (Pirmohamed et al., 2004).

Actualmente, cerca de cien países tienen sistemas nacionales de notificación de RAM a la base de datos del Centro Colaborador de la OMS para la Vigilancia Farmacéutica Internacional. Cuando aparecen señales de problemas relacionados con la seguridad de los medicamentos; la OMS comparte los resultados con todos los países miembros (Ruedy, 2004; Wiholm et al., 2000).

Según la OMS la farmacovigilancia es la prevención y detección de los efectos adversos de los medicamentos. La evaluación atenta de los riesgos y beneficios de los medicamentos se aplica en todas las etapas del ciclo de vida de estos, desde antes de su aprobación hasta su uso por los pacientes (Ruedy, 2004). 
En estudios recientes se ha observado que existe una predisposición genética que aumenta la susceptibilidad de un sujeto para padecer RAM (Kanneh, 2011; Phillips, 2001; IngelmanSundberg, 2001; Ingelman Sundberg, 1999).

Los eventos específicos que han sido reportados indican que las reacciones adversas dermatológicas (RAD) son las más frecuentes en todas las poblaciones sin distinción etaria ni de género y que son las que más obligan al cese del tratamiento (Castro-Pastrana, 2011; Gruchalla, 2000; Unfried, 2008). Dentro de sus subtipos algunas series han reportado la urticaria como principal manifestación clínica de este tipo de RAM, sin embargo, otros han encontrado principalmente al exantema. Respecto a su causalidad es claro que se debe a los antibióticos en gran parte, pero con diferencias al grupo farmacológico responsable, ya que los pacientes en dichas muestras se encontraron en su mayoría polimedicados; otros medicamentos causales incluyen antineoplásicos, anticonvulsivantes y antiinflamatorios no esteroideos (AINES). Las RAD son seguidas en algunas series por la hipoglicemia y en otras por las gastritis agudas, pero encontrándose estas dos entre las primeras causas (Castro-Pastrana, 2011; Gruchalla, 2000; Chan y Nicklason, 2001).

En nuestro país han sido pocas las series donde se documenten las RAM y su incidencia en la población. Se cuenta con un estudio realizado en Pereira en el 2007 que reveló los grupos farmacológicos más frecuentemente relacionados con RAM en el servicio de urgencias, indicando que los antidiabéticos y la warfarina fueron asociados en definitiva en la mayoría de casos con la aparición de efectos no deseados, los cuales fueron respectivamente la hipoglucemia y hemorragias (Pirmohamed et al., 2004). Lo anterior concuerda con la literatura americana, donde en un informe de casos anuales en Estados Unidos de 2006 que incluyó 21,286 reportes, se llegó a la misma conclusión respecto a fármacos como a efectos adversos (Castro-Pastrana, 2011).

\section{Materiales y métodos}

Se realizó un estudio descriptivo longitudinal retrospectivo, llevado a cabo en pacientes hospitalizados en un hospital de segundo nivel de complejidad de Itagüí, Antioquia, quienes recibieron terapia farmacológica para su patología de base entre los años 2010 y 2012. Ingresaron al estudio 589 pacientes, de los cuales solo 507 cumplieron los criterios de inclusión; la información utilizada para este trabajo, fue captada a partir de los registros del programa de farmacovigilancia del hospital.

El análisis estadístico de los datos se realizó mediante el programa G - stat versión 2.0. La asociación entre las variables cualitativas se estableció con la prueba de $\mathrm{Chi}^{2}$ de independencia y para las variables cuantitativas se recurrió a la prueba de T-student.

\section{Resultados}

Desde el año 2010 hasta el primer semestre de 2012, fueron evaluados un total de 507 pacientes en un hospital de segundo nivel de complejidad en Itagüí, Antioquia, que presentaban alguna reacción adversa a medicamentos. En el estudio el $52.07 \%$ corresponde al sexo femenino en comparación con el $47.93 \%$ de sexo masculino. La edad promedio de los pacientes fue de 56.37 años, mientras que la edad en la 
que más se presentaron reacciones adversas fue a los 65 años. Ver tablas 1 y 2 .

Tabla 1. Reacciones adversas según el género. N: Número absoluto de pacientes y \%: porcentaje de pacientes que pertenecientes a cada género que presentaron reacción adversa frente a algún medicamento administrado para su patología de base durante la estadía en el hospital.

\begin{tabular}{|c|c|c|}
\hline Sexo & N & $\%$ \\
\hline Femenino & 264 & 52.07 \\
\hline Masculino & 243 & 47.93 \\
\hline Total & 507 & 100 \\
\hline
\end{tabular}

Fuente. Elaboración propia.

Tabla 2. Medidas de resumen para la edad. La tabla indica la edad en la que fueron más frecuentes las RAM.

\begin{tabular}{|c|c|}
\hline Medida estadística & Edad en años \\
\hline Media & 56.37 \\
\hline Moda & 65 \\
\hline Mediana & 61 \\
\hline
\end{tabular}

Fuente. Elaboración propia.

En cuanto a las reacciones adversas dermatológicas se pudo determinar que la reacción más común dentro de los pacientes evaluados fue el rash cutáneo con un $53.51 \%$ de pacientes, seguida por flebitis química $18.68 \%$, toxicodermia $13.74 \%$, prurito $3.4 \%$. Las demás manifestaciones (síndrome de Steven Jhonson, síndrome del hombre rojo, etc.) tuvieron un porcentaje menor de participación en un $8.95 \%$. De las manifestaciones hematológicas, la más presentada por parte de los pacientes fue la sobreanticoagulación en un $60.19 \%$, seguida de eosinofilia con un $13.12 \%$, el resto de las manifestaciones se presentaron en un menor porcentaje.

La afección más comúnmente presentada por los pacientes en relación con otros sistemas fue la hepatotoxicidad con un porcentaje de $3.19 \%$, seguida de las demás afecciones como: hipoglicemia, nefrotoxicidad, diarrea, emesis entre otras. La información anterior se resume en las tablas 3,4 y 5 .
Tabla 3. Reacciones adversas en piel.

\begin{tabular}{|l|c|c|}
\hline \multicolumn{1}{|c|}{ Manifestación } & Frecuencia & $\%$ \\
\hline Rash & 100 & 53.51 \\
\hline Flebitis química & 34 & 18.68 \\
\hline Toxicodermia & 25 & 13.74 \\
\hline Prurito & 7 & 3.4 \\
\hline Urticaria & 3 & 1.65 \\
\hline S. Steven Jhonson & 2 & 1.10 \\
\hline S. hombre rojo & 2 & 1.10 \\
\hline Otros & 13 & 6.75 \\
\hline Total & 186 & 99.93 \\
\hline
\end{tabular}

Fuente. Elaboración propia.

Tabla 4. Reacciones adversas en Sistema hematológico.

\begin{tabular}{|l|c|c|}
\hline \multicolumn{1}{|c|}{ Medicamentos } & Frecuencias & $\%$ \\
\hline Sobreanticuagulación & 124 & 60.19 \\
\hline Eosinofilia & 27 & 13.12 \\
\hline Leucopenia & 20 & 9.92 \\
\hline Pancitopenia & 11 & 5.34 \\
\hline Neutropenia & 6 & 2.92 \\
\hline Anafilaxia & 4 & 1.94 \\
\hline Trombocitopenia & 4 & 1.95 \\
\hline Otros & 9 & 4.41 \\
\hline Total & 205 & 99.79 \\
\hline
\end{tabular}

Fuente. Elaboración propia.

Tabla 5. Reacciones adversas en otros sistemas.

\begin{tabular}{|l|c|c|}
\hline \multicolumn{1}{|c|}{ Otras } & Frecuencias & \% \\
\hline Hepatotoxicidad & 13 & 13.48 \\
\hline Hipoglicemia & 8 & 8.79 \\
\hline Nefrotoxicidad & 7 & 7.69 \\
\hline Diarrea & 6 & 6.59 \\
\hline Emesis & 4 & 4.40 \\
\hline Otras & 52 & 58.24 \\
\hline Total & 90 & 100 \\
\hline
\end{tabular}

Fuente. Elaboración propia.

En las tablas $6,7,8$ y 9 se muestran los medicamentos que mayor número de RAM produjeron, partiendo de lo siguiente: el antibiótico que más reacción adversa produjo fue la claritromicina con un $15.28 \%$, seguido de la vancomicina con un $9.03 \%$, el meropenem con 
un $8.33 \%$ y el resto de los antibióticos con un porcentaje menor. El analgésico que más se relaciona con la producción de reacciones adversas fue la dipirona con un $56.86 \%$, seguido del tramadol con un $22.55 \%$, acetaminofén $7.84 \%$ y otros analgésicos implicados en un $12.74 \%$ de todos los casos. De los medicamentos anticoagulantes que causaron reacciones adversas a medicamentos, la warfarina produjo el $98.77 \%$ de los casos, el $1.2 \%$ restante lo ocasionaron otros anticoagulantes. De la clasificación de los otros medicamentos implicados, el que produjo más reacciones adversas fue el bisacodilo con un $6.3 \%$, seguido de los demás medicamentos con un menor porcentaje. El lugar de mayor ocurrencia de reacciones medicamentosas, se produjo en el ámbito intrahospitalario, con un porcentaje del $65.74 \%$, el cual corresponde a un total de 284 pacientes de los 507 evaluados. El $34.36 \%$ restante presentó reacciones adversas a nivel extrahospitalario, ver tabla 10. Finalmente, en la tabla 11 se muestra que del total de los 507 pacientes incluidos en el estudio un $88.51 \%$ presentó polimedicación, lo cual corresponde a 439 pacientes y solo el $11.49 \%$ no presentaba polimedicación.

Tabla 6. Medicamentos antibióticos que producen reacciones adversas.

\begin{tabular}{|l|c|c|}
\hline \multicolumn{1}{|c|}{ Medicamento } & Frecuencias & $\%$ \\
\hline Claritromicina & 22 & 15.28 \\
\hline Vancomicina & 13 & 9.03 \\
\hline Meropenem & 12 & 8.33 \\
\hline Ciprofloxacina & 9 & 6.25 \\
\hline Amikacina & 9 & 6.25 \\
\hline Rifampicina & 9 & 6.25 \\
\hline Cefazolina & 9 & 6.25 \\
\hline Oxacilina & 7 & 4.86 \\
\hline Otros & 53 & 33.9 \\
\hline
\end{tabular}

Fuente. Elaboración propia.
Tabla 7. Medicamentos analgésicos que producen reacciones adversas.

\begin{tabular}{|l|c|c|}
\hline \multicolumn{1}{|c|}{ Medicamento } & Frecuencia & $\%$ \\
\hline Dipirona & 58 & 56.86 \\
\hline Tramadol & 23 & 22.55 \\
\hline Acetaminofén & 8 & 7.84 \\
\hline Otros & 13 & 12.74 \\
\hline Total & 102 & 100 \\
\hline
\end{tabular}

Fuente. Elaboración propia.

Tabla 8. Medicamentos anticoagulantes que producen reacciones adversas.

\begin{tabular}{|l|c|c|}
\hline \multicolumn{1}{|c|}{ Medicamento } & Frecuencia & $\%$ \\
\hline Warfarina & 122 & 98.77 \\
\hline Nadroparina/Fondaparinox & 2 & 1.62 \\
\hline Total & 124 & 100 \\
\hline
\end{tabular}

Fuente. Elaboración propia.

Tabla 9. Otros medicamentos que producen reacciones adversas.

\begin{tabular}{|l|c|c|}
\hline Medicamentos & Frecuencia & $\%$ \\
\hline Bisacoldilo & 6 & 6.9 \\
\hline Ranitidina & 5 & 5.75 \\
\hline Metoclopramida & 5 & 5.75 \\
\hline Otros & 71 & 81.65 \\
\hline Total & 87 & 100 \\
\hline
\end{tabular}

Fuente. Elaboración propia.

Tabla 10. Lugar de ocurrencia y reporte de las reacciones adversas.

\begin{tabular}{|l|c|c|}
\hline \multicolumn{1}{|c|}{ Lugar } & Frecuencia & $\%$ \\
\hline Intrahospitalario & 284 & 65.74 \\
\hline Extrahospitalario & 148 & 34.26 \\
\hline
\end{tabular}

Fuente. Elaboración propia.

Tabla 11. Pacientes polimedicados.

\begin{tabular}{|l|c|c|}
\hline Polimedicación & Frecuencia & $\%$ \\
\hline Sí & 439 & 88.51 \\
\hline No & 57 & 11.49 \\
\hline Total & 496 & 100 \\
\hline
\end{tabular}

Fuente. Elaboración propia. 


\section{Discusión}

Ya son muchos los estudios que se han realizado en todo el mundo a propósito de los eventos adversos asociados a medicamentos (EAAM), ya que se han convertido en un gran problema de salud pública (Osés et al., 2003; INVIMA, 2003).

En Colombia se ha desarrollado un protocolo con el fin de generar una política de seguridad del paciente, permitiendo cumplir así en teoría la política promulgada por la OMS en la "Alianza mundial para la seguridad del paciente" (World Health Organization, 2002; Aranaz, 2005).

Para esta investigación se tomaron como ejemplo modelos de farmacovigilancia de España y diferentes países latinoamericanos, ya que son países con condiciones y prácticas en salud similares a Colombia, permitiendo una correlación más objetiva.

El estudio ENEAS realizado en el año 2005, recolectó 6500 historias clínicas. En este, después de analizar los datos se concluyó que la población más afectada fue el género femenino en un $53.9 \%$. Además, las RAM más frecuentes fueron las que aparecen en el sistema cutáneo y hematológico, tipo prurito y hemorragia por sobreanticoagulación. Estos datos concuerdan con nuestro estudio en un porcentaje del $52.07 \%$ en el género femenino e igual compromiso de sistemas.

Una observación que llama la atención es que los pacientes atendidos en los diversos centros de referencia de las investigaciones tomadas en cuenta y en nuestro estudio, son pacientes que generalmente reciben múltiples tratamientos farmacológicos, que los exponen a un riesgo elevado de reacciones adversas a medicamentos.

Esta polifarmacia genera un factor de riesgo para RAM; aunque no se realizó su medición y su impacto a nivel extrahospitalario e intrahospitalario en los artículos revisados. En nuestro estudio podemos concluir que la mayor parte de las reacciones adversas presentadas ocurren a nivel intrahospitalario, evento explicado al parecer por el tipo de medicamentos que se utilizan en este nivel; a pesar de que en el ámbito extrahospitalario también se presenta la polifarmacia y por ende las reacciones adversas.

Partiendo de lo anterior sería ideal contar con varios artículos o estudios que nos permitieran determinar el impacto de los efectos adversos en estas áreas de la terapéutica, para así poder comparar el peso relativo de este estudio.

En el estudio el género más afectado fue el femenino con un $52.07 \%$. Hallazgo que puede ser explicado por las diferencias en los aspectos farmacocinéticos como: diferencias metabólicas, la composición corporal, la capacidad de depuración renal y las diferencias hormonales, además de isoenzimas específicas CYTP 450 o CYP3 A 4 que tienen mayor efecto en este género. Adicionalmente, también podría explicarse por factores epidemiológicos (mayor prescripción en unos medicamentos que en otros).

En cuanto a los órganos más afectados con las RAM, no existe un consenso entre nuestro estudio y aquellos trabajos tomados como referencia. Tampoco existe una concordancia entre los resultados de los diferentes estudios. Esto es posiblemente explicado por la diversidad de motivos de consulta atendidos en los centros de salud, su nivel de complejidad y la especialización del centro en el cual se trata una mayor proporción de pacientes quirúrgicos.

En cuanto a la prevalencia de la sintomatología presentada por estos pacientes; también hubo discrepancia entre los diferentes trabajos y el nuestro. Aunque en algunos trabajos, compa- 
rados con el desarrollado en este hospital de segundo nivel en Itagüí, Antioquia, mantienen la incidencia, pero no su porcentaje y orden de prelevancia; es decir, nuestro estudio arrojó que el síntoma de mayor prevalencia en estos pacientes, según el órgano afectado, independiente del sexo y la edad fue para piel rash, seguido de flebitis química y toxicodermia; para el hematológico fue sobreanticoagulación seguido de eosinophilia y leucopenia y en otras manifestaciones, las de mayor prevalencia fueron hepatotoxicidad, hipoglicemia y nefrotoxicidad.

En el resto de los estudios, se mantuvo la incidencia de síntomas como la hipoglucemia, el rash cutáneo, la sobreanticoagulación, entre otros (Informe ENEAS), pero no mantuvo la prevalencia. Los hallazgos mencionados anteriormente, pueden deberse a la polifarmacia a la cual son sometidos estos pacientes y aspectos farmacocinéticos y farmacodinámicos que varía de individuo a individuo.

Para las RAM producidas por los diferentes grupos farmacológicos, se debe aclarar que su presencia está sujeta a patologías presentadas, la edad del paciente, la predisposición genética y antecedentes personales farmacológicos y alérgicos entre otras causas.

Lo más importante es comprender que las RAM constituyen un problema de salud pública que cada día se hace más prevalente en nuestra población. Esta información la podemos confirmar con la base de datos Scielo, donde fue publicado en el año 2006 el artículo «Reacciones adversas en salud pública». En este trabajo la población de estudio correspondió a los pacientes que acudían al servicio de urgencias de una institución hospitalaria en la ciudad de Pereira, Colombia; dicho artículo hace énfasis en las precauciones que se deben tener con la polifarmacia, en especial con los medicamen- tos tipo dipirona, warfarina, metoclopramida, AINES y antibióticos entre otros, asociados con una edad mayor de 55 años, con antecedentes previos de RAM y síntomas en la piel, sistema digestivo y neurológico, ya que estos contribuirían al aumento de la incidencia y muy probablemente de la morbimortalidad.

Por todo lo anterior, es recomendable fortalecer los programas de fármacovigilancia y, reestructurar los ya existentes, con el fin de realizar un mejor control en el desarrollo de las RAM. Esperamos que este estudio logre un gran impacto sobre el personal asistencial para que conozca la gran importancia de adoptar medidas impartidas por estos programas de farmacovigilancia, con el fin de disminuir en lo mayor posible el riesgo y la prevalencia de aparición de RAM.

\section{Agradecimientos}

Agradecemos a la Facultad de Ciencias de la Salud de la Corporación Universitaria Remington y al E.S.E Hospital San Rafael del municipio de Itagüí por generar el ambiente propicio para la ejecución de la presente investigación y por la financiación de la misma.

\section{Referencias}

- Aranaz, J. (2005). Estudio Nacional de Efectos Adversos ligados a la Hospitalización. ENEAS, 169.

- Castro-Pastrana, L. (2011). Cutaneous adverse drug reactions in children: An analysis of reports from the canadian pharmacoge- 
nomics network for drug safety. J Popul ther Clin Pharmacol, 18(1), 106-20.

- Chan, M., y Nicklason, F. (2001). Adverse drug events as a cause of hospital admission in the elderly. Intern Med J, 37(4), 199-205.

- Gruchalla R. S. y Beltrani V. S. (2000). Druginduced cutaneous reactions. En: Leung D. Y. M., Greaves M. W. (Ed.), Allergic Skin Disease: A Multidisciplinary Approach (pp. 307-336). Informa Healthcare.

- Ingelman Sundberg, M. (1999). Polymorphic human cytochrome P450 enzymes: an opportunity for individualized drug treatment. Trends Pharmacol. Sci., 342-349.

- Ingelman-Sundberg, M. (2001). Pharmacogenetics: an opportunity for a safer and more efficient pharmacotherapy. $J$ Intern. Med, 250(3), 186-200.

- INVIMA. (2002). Normatividad Medicamentos 2002.

- Kanneh, A. (2011). Adverse drug reactions: causes, types, pathways and mechanisms. Nursing Children and Young People, 23(4), 23-26.

- Osés I., Burillo-Putze G., Munné P., Nogué S. y Pinillos M.A. (2003). Intoxicaciones medicamentosas (I): Psicofármacos y antiarrítmicos. Anales Sis San, 26(s1), 49-63.
Phillips, K. A., Veenstra, D. L., Oren, E., Lee, J. K. y Sadee, W. (2001). Potential Role of Pharmacogenomics in Reducing Adverse Drug Reactions: A Systematic Review. JAMA, 286(18), 2270-2279. doi:10.1001/ jama.286.18.2270.

Pirmohamed Munir, James Sally, Meakin Shaun, Green Chris, Scott Andrew K., Walley Thomas J. et al. (2004). Adverse drug reactions as cause of admission to hospital: prospective analysis of 18820 patients. British Medical Journal, 329, 15-19.

- Ruedy, J. (2004). Adverse Events: past and Future. CMAJ, 171(6), 549.

- Unfried S. E. (2008). Reacciones adversas a medicamentos como causa de consulta en el servicio de emergencias del Hospital San Juan de Dios. Revista Médica de la Universidad de Costa Rica, 2(1), 39-50.

Wiholm, B. E., Olsson, S., Moore, N. y Waller, P. (2000) Spontaneous Reporting Systems Outside the US. En B. L. Strom. (Ed.), Pharmacoepidemiology (Third Edition). Chichester, UK: John Wiley \& Sons, Ltd. doi: 10.1002/0470842555.ch11

World Health Organization. (2002). The importance of pharmacovigilance. Safety monitoring of medicinal products. Recuperado dehttp://goo.gl/WdliON 